\title{
Multimodality Minimally Invasive Treatment of Large Joint Osteoarthritis: A Pilot Trial of Stromal Vascular Fraction from Autologous Fat Combined with Platelet-Rich Plasma
}

\author{
Philip Giddings, Insiyah Campwala, Shayle Gupta, Christopher Jobe and Subhas Gupta* \\ Department of Plastic Surgery, Loma Linda University School of Medicine, USA
}

Submission: July 19, 2017; Published: July 26, 2017

*Corresponding author: Subhas Gupta, Department of Plastic Surgery, Loma Linda University School of Medicine, Loma Linda, California, 92354, USA, Email: sgupta@LLU.edu

\begin{abstract}
Introduction: Osteoarthritis $(\mathrm{OA})$ is a degenerative joint disease that can cause severe pain in those affected. Currently, there are no therapeutic drugs available to treat osteoarthritis. While total joint arthroplasties (TJA) allow for improved function, there are no less invasive, yet effective procedures that can be done for patients unwilling or unable to undergo major surgery.

Methods: Patients with moderate osteoarthritis presenting with severe hip pain were selected for treatment. After a detailed informed consent process, patients were taken to the operating room for the proposed combination treatment. SVF was isolated using liposuction equipment, and PRP and SVF were centrifuged independently. With ultrasound guidance, a mixture of PRP $(10 \mathrm{~mL})$ and SVF $(10 \mathrm{~mL})$ was injected into the osteoarthritic hip joints of patients under general anesthesia. The Western Ontario and McMaster Universities Arthritis Index (WOMAC) scale was used to assess patients preoperatively and postoperatively to assess pain improvement.
\end{abstract}

Results: WOMAC scores were 67/96 preoperatively, and 17/96 at five weeks post-op. This indicated a decrease in pain and increased mobility. The procedure was both efficient-taking approximately one hour of operative time-and cost effective. No intraoperative or postoperative morbidities were found.

Conclusion: This new combination therapy offers a minimally invasive, low-risk and high-satisfaction treatment for osteoarthritic joints.

Abbreviations: OA: Osteoarthritis; TJA: Total Joint Arthroplasties; PRP: Platelet-rich plasma; SVF: Stromal Vascular Fraction; WOMAC: Western Ontario and McMaster Universities Osteoarthritis Index; MSC: Mesenchymal Stem Cells; HA: Hyaluronic Acid

\section{Introduction}

Osteoarthritis $(\mathrm{OA})$ is a degenerative joint disease that can cause severe pain in those affected. While the quantity of pain may vary from mild to severe, the negative effects of this ailment can extend beyond the boundaries of pain and also affect workcapability and lifestyle. Currently, there are no therapeutic drugs available to treat $\mathrm{OA}$. While total joint arthroplasties (TJA) can allow for improved symptoms, there are no lessinvasive yet affective procedures that can be done for patients unwilling or unable to undergo major surgery as well as those whose symptoms have not reached adequate severity to qualify for TJA. Platelet-rich plasma (PRP) has been shown to reduce the symptoms of $\mathrm{OA}$ when injected into the affected joint. A systematic review of this established orthopedic procedure has shown very positive results. On average, Western Ontario and
McMaster Universities Osteoarthritis Index (WOMAC) scores decreased significantly $(\mathrm{P}=.0008)$ after treatment with PRP [1].

Patients treated by this method alone have improved selfreported pain (at least a $50 \%$ reduction from original value) as well as improved lower extremity function [2]. While the effects of PRP treatment of OA have been positive, treatment with SVF offers additional benefits that PRP alone cannot. Subcutaneous fat has an abundant reservoir of adipose-derived stem cells, growth factors, and other regenerative cell types that can be isolated from the stromal vascular fraction (SVF). SVF contains a significant amount of mesenchymal stem cells (MSC) which have great potential in the field of regenerative medicine [3]. The potential to generate new cartilage in osteoarthritic joints goes beyond treatment of the symptoms and offers a reversal of the harmful pathology. 
Preclinical research has been done in several animal studies in order to demonstrate the benefit and also safety of treating osteoarthritic joints with SVF [4]. In more recent research, SVF treatment has been done in over 1700 patients with $\mathrm{OA}$. The results have been overwhelmingly positive with a $75 \%$ score improvement in $63 \%$ of patients and at least a $50 \%$ improvement in $91 \%$ of all patients [5]. Treatment of OA with combined therapy of stromal-vascular fraction (SVF) and platelet-rich plasma (PRP) holds the potential to provide a lessinvasive treatment of $\mathrm{OA}[2,6]$. This may postpone the need for TJA. There are several complications associated with TJA such as thromboembolism, myocardial infarction, stroke, and risk of infections $[7,8]$. Delaying or eliminating the need for TJA will serve to minimize these potential risks. Considering the positive results seen from both SVF and PRP treatment, the reduced risk of surgical complication when compared to TJA, and the possibility for equal or greater pain improvement and joint functionality compared to TJA, we believe it would be advantageous to investigate a combined PRP and SVF therapy for relief of $\mathrm{OA}$.

\section{Methods}

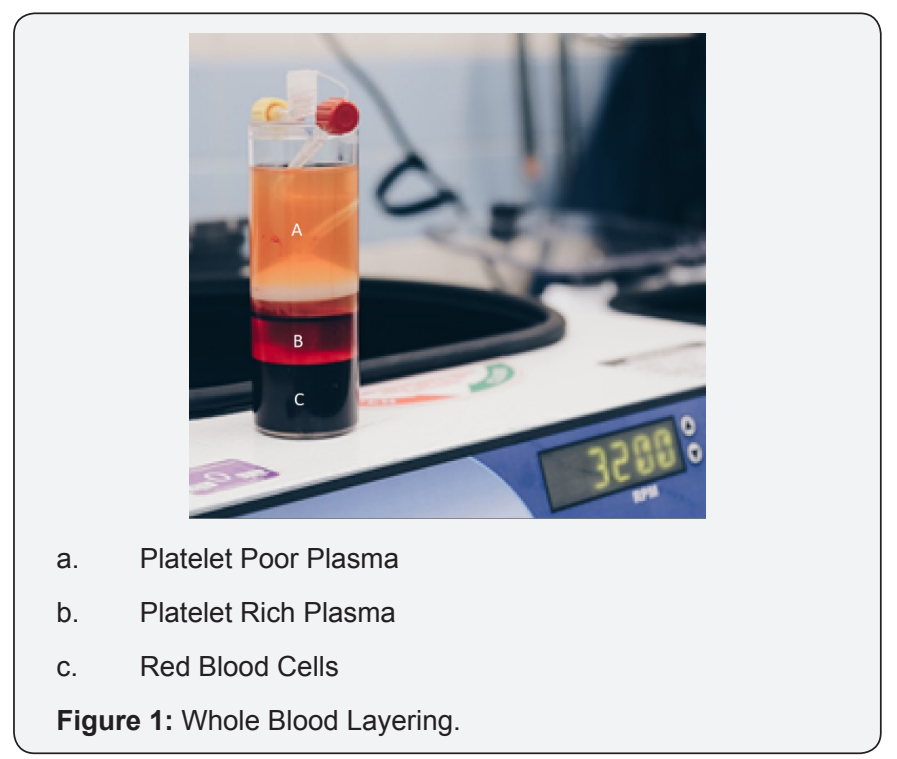

An index patient with moderate osteoarthritis presenting with severe hip pain was selected for treatment. X-ray radiographic grading was used for hip arthritis diagnosis. After a detailed informed consent process, the 61 year old male was taken to the operating room for the proposed combination treatment. $180 \mathrm{~mL}$ of tumescent were injected into the abdomen of the patient under general anesthesia. $60 \mathrm{~mL}$ of whole blood was taken from the left arm and combined with $40 \mathrm{~mL}$ of heparin. The $100 \mathrm{~mL}$ solution was centrifuged in the BioCueTM Platelet Concentration System (Biomet $\AA$ Biologics, Warsaw, Indiana) on $3200 \mathrm{rpm}$ for 15 minutes. PRP (10cc) was isolated from the middle layer of the whole blood (Figure 1). SVF was isolated using a 3mL liposuction cannula and 60cc syringe via an incision made below the umbilicus. The $60 \mathrm{~mL}$ total harvest from the abdomen was separated into $5 \mathrm{cc}$ syringes and centrifuged for $5 \mathrm{~min}$. SVF (10cc) was isolated from the second layer of each syringe (Figure 2). A 5-0 fast gut suture was used to close the small abdominal incision. An 18 gauge spinal needle was used to inject $10 \mathrm{cc}$ of saline into the hip joint. With ultrasound guidance, a mixture of PRP $(10 \mathrm{~mL})$ and SVF $(10 \mathrm{~mL})$ was injected into the osteoarthritic hip joints using an 18 gauge spinal needle (Figure 3). A dressing was placed on the injection site as dressing. The entire procedure took approximately one hour. The Western Ontario and McMaster Universities Arthritis Index (WOMAC) scale was used to assess patients preoperatively and postoperatively to measure pain, stiffness, and physical function improvement.

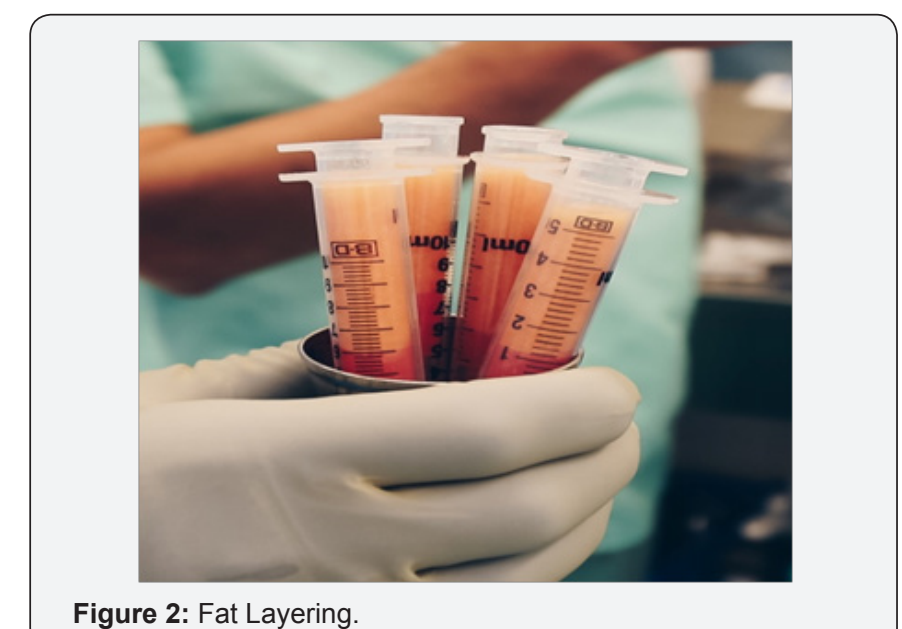

Figure 2: Fat Layering.

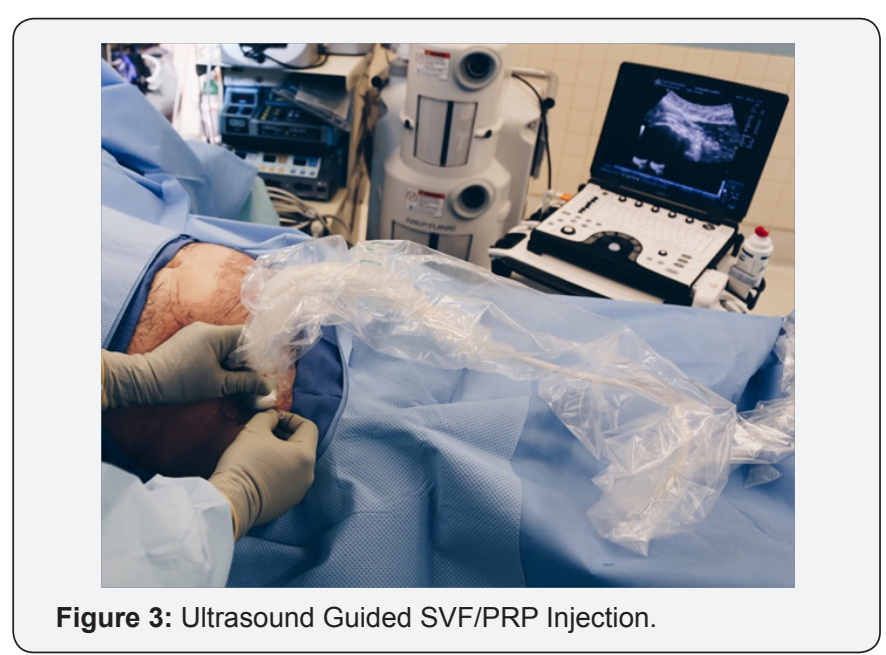

Results

WOMAC scores were 67/96 preoperatively, and 17/96 at five weeks post-op. This indicated a decrease in pain and increased mobility. The procedure was both efficient-taking approximately one hour of operative time-and cost effective. No intraoperative or postoperative morbidities were found. 


\section{Discussion}

The Centers for Disease Control and Prevention estimates that osteoarthritis affects over 30 million adults in the United States [9]. This new combination treatment holds the potential to become a standard of care. Pak et al conducted a similar analysis in 3 osteoarthritic knees in Korea-in which they injected SVF, hyaluronic acid (HA), and PRP activated by calcium chloride. For three weeks post initial injection of SVF, HA, and PRP, they reinjected weekly with additional PRP. MRI data showed cartilage-like tissue regeneration with this procedure [10].

Another study from Vietnam found that SVF/PRP injection with arthroscopic micro fracture treatment resulted in significantly reduced pain and WOMAC scores when compared to those who received arthroscopic micro fracture in the absence of SVF/PRP [11]. As demonstrated by the change in WOMAC scores in our pilot study, the SVF/PRP combination therapy provided substantial pain and stiffness relief for patients and led to an increase in physical function. It is also minimally invasive and cost-effective: total joint arthroplasty costs $\$ 68,400$ while SVF/ PRP treatment costs $\$ 6,400$ at our institution. Improved pain control may also be associated with decreased complications, reduced length of hospital stay, improved patient comfort and satisfaction, less time off work, and reduced healthcare costs. This treatment can also lead to greater mobility in osteoarthritic joints.

\section{Conclusion}

This new combination therapy offers a minimally invasive, low-risk and high-satisfaction treatment for osteoarthritic joints. Future work will be focused on expanding our sample size and ideal SVF and PRP dose determination.

\section{References}

1. Meheux CJ, McCulloch PC, Lintner DM, Varner KE, Harris JD (2016) Efficacy of Intra-articular Platelet-Rich Plasma Injections in Knee
Osteoarthritis: A Systematic Review. Arthroscopy 32(3): 496-505.

2. Montañez-Heredia, Irízar S, Huertas PJ, Otero E, Del Valle M, et al. (2016) Intra-Articular Injections of Platelet-Rich Plasma versus Hyaluronic Acid in the Treatment of Osteoarthritic Knee Pain: A Randomized Clinical Trial in the Context of the Spanish National Health Care System. Int J Mol Sci 17(7).

3. Hematti P, Keating A (2013) Mesenchymal stromal cells in regenerative medicine: A Perspective. Mesenchymal Stromal Cells. Biology and clinical applications, p. 3-16.

4. Paterson L, Nicholls M, Bennell L, Bates D (2016) Intra-articular injection of photo-activated platelet-rich plasma in patients with knee osteoarthritis: a double-blind, randomized controlled pilot study. BMC MusculoskeletDisord 17: 67.

5. Michalek J, Moster R, Lukac L, Proefrock K, Petrasovic M, et al. (2015) Autologous adipose tissue-derived stromal vascular fraction cells application in patients with osteoarthritis. Cell Transplant.

6. Gibbs N, Diamond R, Sekyere EO, Thomas WD (2015) Management of knee osteoarthritis by combined stromal vascular fraction cell therapy, platelet-rich plasma, and musculoskeletal exercises: a case series. J Pain Res 8: 799-806.

7. Eriksson BI, Borris LC, Friedman RJ, Haas S, Huisman MV, et al. (2008) Rivaroxaban versus enoxaparin for thromboprophylaxis after hip arthroplasty. N Engl J Med 358(26): 2765-2675.

8. Schrama JC, Espehaug B, Hallan G, Engesaeter LB, Furnes O, et al. (2010) Risk of revision for infection in primary total hip and knee arthroplasty in patients with rheumatoid arthritis compared with osteoarthritis: a prospective, population-based study on 108,786 hip and knee joint arthroplasties from the Norwegian Arthroplasty Register. Arthritis Care Res 62(4): 473-479.

9. Osteoarthritic Fact Sheet (2017) Centers for Disease Control and Prevention, USA.

10. Pak J, Lee JH, Park KS, Jeong BC, Lee SH (2016) Regeneration of cartilage in human knee osteoarthritis with autologous adipose tissuederived stem cells and autologous extracellular matrix. BioRes Open Access 5(1): 192-200.

11. Nguyen PD, Tran TD, Nguyen HT, Vu HT, Le PT, et al. (2017) Comparative Clinical Observation of Arthroscopic Microfracture in the Presence and Absence of a Stromal Vascular Fraction Injection for Osteoarthritis. Stem Cells Translational Medicine 6(1): 187-195.

\section{Your next submission with Juniper Publishers will reach you the below assets}

- Quality Editorial service

- Swift Peer Review

- Reprints availability

- E-prints Service

- Manuscript Podcast for convenient understanding

- Global attainment for your research

- Manuscript accessibility in different formats

( Pdf, E-pub, Full Text, Audio)

- Unceasing customer service

Track the below URL for one-step submission https://juniperpublishers.com/online-submission.php 